PROCEEDINGS OF THE

AMERICAN MATHEMATICAL SOCIETY

Volume 138, Number 9, September 2010, Pages 3293-3299

S 0002-9939(10)10350-5

Article electronically published on April 16, 2010

\title{
A LOWER BOUND ON THE SUBRIEMANNIAN DISTANCE FOR HÖLDER DISTRIBUTIONS
}

\author{
SLOBODAN N. SIMIĆ
}

(Communicated by Bryna Kra)

\begin{abstract}
Whereas subriemannian geometry usually deals with smooth horizontal distributions, partially hyperbolic dynamical systems provide many examples of subriemannian geometries defined by non-smooth (namely, Hölder continuous) distributions. These distributions are of great significance for the behavior of the parent dynamical system. The study of Hölder subriemannian geometries could therefore offer new insights into both dynamics and subriemannian geometry. In this paper we make a small step in that direction: we prove a Hölder-type lower bound on the subriemannian distance for Hölder continuous nowhere integrable codimension one distributions. This bound generalizes the well-known square root bound valid in the smooth case.
\end{abstract}

\section{INTRODUCTION}

The purpose of this paper is to prove a lower bound in terms of the Riemannian distance for subriemannian geometries defined by codimension one distributions that are only Hölder continuous, generalizing the well-known square root bound valid in the smooth case.

A subriemannian or Carnot-Carathéodory geometry is a pair $(M, H)$, where $M$ is a smooth manifold and $H$ is a nowhere integrable distribution (a notion we will define shortly) endowed with a Riemannian metric $g$. Classically, both $H$ and $g$ are assumed to be smooth, e.g., of class $C^{\infty} ; H$ is usually called a horizontal distribution (or a polarization, by Gromov Gro96) and is taken to be bracket-generating (see below for a definition). Piecewise smooth paths a.e. tangent to $H$ are called horizontal paths. The subriemannian distance is defined by

$$
d_{H}(p, q)=\inf \{|\gamma|: \gamma \text { is a horizontal path from } p \text { to } q\},
$$

where the length $|\gamma|$ of a horizontal path $\gamma: I \rightarrow M$ is defined in the usual way, $|\gamma|=\int_{I} g(\dot{\gamma}(t), \dot{\gamma}(t))^{1 / 2} d t$

Recall that a smooth distribution $H$ is called bracket generating if any local smooth frame $\left\{X_{1}, \ldots, X_{k}\right\}$ for $H$, together with all of its iterated Lie brackets, spans the whole tangent bundle of $M$. (In PDEs, the bracket-generating condition is also called the Hörmander condition.) By the well-known theorem of Chow and Rashevskii [Mon02, if $H$ is bracket generating, then $d_{H}(p, q)<\infty$, for all $p, q \in M$.

Received by the editors June 29, 2009 and, in revised form, December 18, 2009 and December 23, 2009.

2010 Mathematics Subject Classification. Primary 51F99, 53 B99.

Key words and phrases. Distribution, Hölder continuity, subriemannian distance.

(C)2010 American Mathematical Society

Reverts to public domain 28 years from publication 
However, $H$ does not have to be bracket generating, or even smooth, to define a reasonable subriemannian geometry. Important examples of such distributions come from dynamical systems. Let $f: M \rightarrow M$ be a $C^{r}(r \geq 1)$ partially hyperbolic diffeomorphism of a smooth compact Riemannian manifold $M$. This means that $f$ is like an Anosov diffeomorphism, but in addition to hyperbolic behavior (i.e., exponential contraction and exponential expansion), $f$ also exhibits weakly hyperbolic or non-hyperbolic behavior along certain tangent directions. More precisely, $f$ is partially hyperbolic if the tangent bundle of $M$ splits continuously and invariantly into the stable, center, and unstable bundles (i.e., distribution)

$$
T M=E^{s} \oplus E^{c} \oplus E^{u},
$$

such that the tangent map $T f$ of $f$ exponentially contracts $E^{s}$, exponentially expands $E^{u}$ and this hyperbolic action on $E^{s} \oplus E^{u}$ dominates the action of $T f$ on $E^{c}$ (see Pes04, PS04). The stable and unstable bundle are always uniquely integrable, giving rise to the stable and unstable foliations, $W^{s}, W^{u}$.

A partially hyperbolic diffeomorphism is (rather unfortunately) called accessible if every two points of $M$ can be joined by an su-path, i.e., a continuous piecewise smooth path consisting of finitely many arcs, each lying in a single leaf of $W^{s}$ or a single leaf of $W^{u}$. If $f$ is accessible, then the distribution $E^{s} \oplus E^{u}$ is clearly non-integrable. Dolgopyat and Wilkinson DW03. proved that in the space of $C^{r}$ $(r \geq 1)$ partially hyperbolic diffeomorphisms there is a $C^{1}$ open and dense set of accessible diffeomorphisms. Since invariant distributions of a partially hyperbolic diffeomorphism are in general only $C^{\theta}$, for some $\theta \in(0,1)$ (see [HPS77, Pes04]), this result shows that there is an abundance of subriemannian geometries defined by non-smooth, Hölder continuous distributions. Since these distributions play a crucial role in the dynamics of partially hyperbolic systems and the accessibility property is frequently key to ergodicity (cf., e.g., PS04), a better understanding of subriemannian geometries defined by Hölder distributions could be very useful in dynamical systems. This paper provides a small initial step in that direction.

When $H$ is not smooth, the definition of nowhere integrability is more subtle than in the smooth case. One should keep in mind the following example (we thank an anonymous referee for bringing it up): suppose $H$ is smooth and integrable in a neighborhood of a point $p$ and non-integrable elsewhere. Since every two points can still be connected by a horizontal path, $d_{H}$ is finite everywhere. Let $L$ be the leaf through $p$ of the local foliation of $U$ tangent to $H$. Then unless $q$ lies in $L, d_{H}(p, q)$ is bounded away from zero by a uniform constant, making the subriemannian distance discontinuous with respect to the Riemannian distance for any Riemannian metric on $M$. See Figure 1 .

The following definition prohibits this type of behavior.

Definition. A distribution $H$ on $M$ is called nowhere integrable if for every $p \in M$ and every $\varepsilon>0$, there exists a neighborhood $U$ of $p$, such that any point in $U$ can be connected to $p$ by a horizontal path of length $<\varepsilon$.

From now on, we will assume that the Riemannian metric on $H$ is the restriction of a Riemannian metric from the ambient manifold $M$. If $H$ is a smooth bracketgenerating distribution, the relation between the subriemannian distance $d_{H}$ and the Riemannian distance, which we denote by dist, is well understood and is characterized by the Ball-Box theorem [Mon02, Gro96. When $H$ is of codimension one and bracket-generating, this theorem states that in the vertical direction, i.e., along 


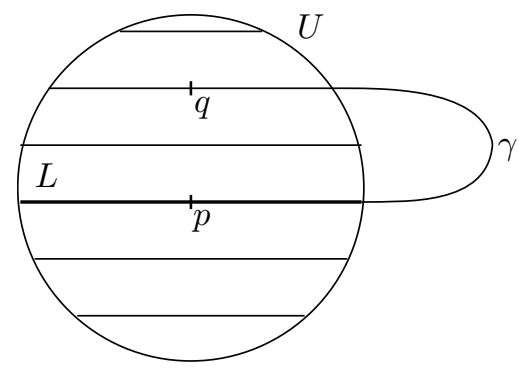

Figure 1. Every horizontal path $\gamma$ connecting $p$ and $q$ has to leave $U$.

any short smooth path $\gamma$ transverse to $H, d_{H}$ is equivalent to $\sqrt{\text { dist. }}$ That is, there exist constants $a, b>0$ such that for all $p, q$ on $\gamma$,

$$
a \sqrt{\operatorname{dist}(p, q)} \leq d_{H}(p, q) \leq b \sqrt{\operatorname{dist}(p, q)} .
$$

In the horizontal direction, i.e., along any horizontal path, $d_{H}$ and dist are clearly equivalent. This means that subriemannian geometry is non-isotropic: it behaves differently in different directions. Subriemannian spheres are far from being "round". In the Heisenberg group, for instance, subriemannian spheres look like an apple Mon02.

In Gro96, M. Gromov gave a short and elegant proof (without details) of the lower bound for $d_{H}$, i.e., the left hand side of (1). His proof uses the assumption that $H$ is $C^{1}$ only in the following way: if $\alpha$ is a 1 -form such that $\operatorname{Ker}(\alpha)=H$, then

$$
\left|\int_{\partial D} \alpha\right| \leq K|D|
$$

for every $C^{1}$ immersed 2-disk with piecewise $C^{1}$ boundary, where $K$ is a constant independent of $D$. Here, $|D|$ denotes the area of $D$. If $H$ (hence $\alpha$ ) is $C^{1}$, this follows directly from the Stokes theorem.

Gromov also remarks that without the $C^{1}$ assumption, the square root estimate probably fails. We will show that this is indeed the case, in the sense that if $H$ is only $C^{\theta}$, for some $0<\theta<1$, and nowhere integrable, then in the vertical direction, $d_{H}(p, q) \geq C \operatorname{dist}(p, q)^{1 /(1+\theta)}$. To generalize Gromov's approach to $C^{\theta}$ horizontal distributions, one needs a generalization of the estimate (2) to forms $\alpha$ that are only Hölder. One such estimate was recently proved in Sim09 and states that the integral of a $C^{\theta} k$-form $\alpha$ over the boundary of a sufficiently small $(k+1)$-disk $D$ is bounded by a certain multiplicative convex combination of the $(k+1)$-volume $|D|$ of $D$ and the $k$-dimensional area $|\partial D|$ of its boundary; see Theorem 2.1] in the next section.

Our main result is the following:

Theorem. Suppose that $H$ is a nowhere integrable codimension one distribution of class $C^{\theta}$, for some $0<\theta<1$, on a smooth compact Riemannian manifold $M$. Assume that the Riemannian metric on $H$ is the restriction of the ambient metric from $M$. Then there exists a constant $\varrho>0$ such that for any $C^{1}$ path $\gamma$ transverse to $H$ and for every two points $p, q$ on $\gamma$ with Riemannian distance less than $\varrho$, we have

$$
d_{H}(p, q) \geq C \operatorname{dist}(p, q)^{\frac{1}{1+\theta}}
$$


where $C>0$ is a constant that depends only on $H$ and $\gamma$.

Remark. (a) Observe that if $\operatorname{dist}(p, q)=\varepsilon$ is small, then $\varepsilon^{1 / 2} \gg \varepsilon^{1 /(1+\theta)}$, which means that the lower bound on $d_{H}(p, q)$ is tighter for $C^{1}$ distributions than for $C^{\theta}$ ones.

(b) It is clear that in any horizontal direction, $d_{H}$ is equivalent to dist.

\section{Auxiliary Results}

The main tool in the proof will be the following inequality.

2.1. Theorem (Theorem A, Sim09]). Let $M$ be a compact manifold and let $\alpha$ be a $C^{\theta} k$-form on $M$, for some $0<\theta<1$ and $1 \leq k \leq n-1$. There exist constants $\sigma, K>0$, depending only on $M, \theta$, and $k$, such that for every $C^{1}$-immersed $(k+1)$ disk $D$ in $M$ with piecewise $C^{1}$ boundary satisfying $\max \{\operatorname{diam}(\partial D),|\partial D|\}<\sigma$, we have

$$
\left|\int_{\partial D} \alpha\right| \leq K\|\alpha\|_{C^{\theta}}|\partial D|^{1-\theta}|D|^{\theta}
$$

As before, $|D|$ denotes the $(k+1)$-volume of $D$, and $|\partial D|$ denotes the $k$-volume (or area) of its boundary.

Remark. If $k=1$, then $\operatorname{diam}(\partial D) \leq|\partial D|$, so the assumption $\operatorname{diam}(\partial D)<\sigma$ is superfluous.

The Hölder norm of $\alpha$ on $M$ is defined in a natural way as follows. Let $\mathscr{A}=$ $\{(U, \varphi)\}$ be a finite $C^{\infty}$ atlas of $M$. We say that $\alpha$ is $C^{\theta}$ on $M$ if $\alpha$ is $C^{\theta}$ in each chart $(U, \varphi)$, i.e., if $\left(\varphi^{-1}\right)^{*} \alpha$ is $C^{\theta}$, for each $(U, \varphi) \in \mathscr{A}$. We set

$$
\|\alpha\|_{C^{\theta}}=\max _{(U, \varphi) \in \mathscr{A}}\left\|\left(\varphi^{-1}\right)^{*} \alpha\right\|_{C^{\theta}(\varphi(U))} .
$$

For a $C^{\theta}$ form $\alpha=\sum a_{I} d x_{I}$ defined on an open subset $U \subset \mathbb{R}^{n}$, we set

$$
\|\alpha\|_{C^{\theta}(U)}=\max _{I}\left\|a_{I}\right\|_{C^{\theta}}
$$

and for a bounded function $f: U \rightarrow \mathbb{R}$,

$$
\|f\|_{C^{\theta}(U)}=\|f\|_{\infty}+\sup _{x \neq y} \frac{|f(x)-f(y)|}{|x-y|^{\theta}} .
$$

We will also need the following version of the solution to the isoperimetric problem "in the small".

2.2. Lemma (Gro83, Sublemma 3.4. $\mathrm{B}^{\prime}$ ). For every compact manifold $M$, there exists a small positive constant $\delta_{M}$ such that every $k$-dimensional cycle $Z$ in $M$ of volume less than $\delta_{M}$ bounds a chain $Y$ in $M$, which is small in the following sense:

(i) $|Y| \leq c_{M}|Z|^{(k+1) / k}$, for some constant $c_{M}$ depending only on $M$.

(ii) The chain $Y$ is contained in the $\varrho$-neighborhood of $Z$, where $\varrho \leq c_{M}|Z|^{1 / k}$.

The following corollary is immediate.

2.3. Corollary. If $\Gamma$ is a closed piecewise $C^{1}$ path in a compact manifold $M$ with $|\Gamma|<\delta_{M}$, then there exists a 2-disk $D \subset M$ such that $\partial D=\Gamma$,

$$
|D| \leq c_{M}|\Gamma|^{2},
$$

and $D$ is contained in the $\varrho$-neighborhood of $\Gamma$, where $\varrho \leq c_{M}|\Gamma|$. 


\section{Proof of the theorem}

We follow Gromov's proof in Gro96, p. 116. Since the statement is local and concerns arbitrary directions transverse to $H$, we can assume without loss of generality that $\gamma$ is a unit speed Riemannian geodesic.

Also without loss, we can assume that $H$ is transversely orientable. If not, pass to a double cover of $M$. Let $X$ be a unit vector field everywhere orthogonal to $H$ and define a 1-form $\alpha$ on $M$ by

$$
\operatorname{Ker}(\alpha)=H, \quad \alpha(X)=1
$$

Define another 1-form $\alpha_{\gamma}$ on $M$ by requiring that

$$
\operatorname{Ker}\left(\alpha_{\gamma}\right)=H, \quad \alpha_{\gamma}(\dot{\gamma})=1
$$

Since $H$ is $C^{\theta}$, so are $\alpha$ and $\alpha_{\gamma}$. (It is reasonable to think of $\|\alpha\|_{C^{\theta}}$ as the Hölder norm of $H$.) Since $\alpha$ and $\alpha_{\gamma}$ have the same kernel, there exists a function $\lambda$ such that $\alpha=\lambda \alpha_{\gamma}$. Writing $\dot{\gamma}=c X+w$, for some $w \in H$ and taking the Riemannian inner product with $X$, we obtain $\lambda=c=\cos \varangle(\dot{\gamma}, X)=\sin \phi$, where $\phi$ is the angle between $\dot{\gamma}$ and $H$. Evaluating both sides of $\alpha=\lambda \alpha_{\gamma}$ at $\dot{\gamma}$, we see that $\lambda=\sin \phi$. Thus $\alpha=\sin \phi \alpha_{\gamma}$. Set $\phi_{0}=\min \phi$. By assumption $\phi_{0}>0$.

Let $\tau=\min \left(\delta_{M}, \sigma\right)$, where $\delta_{M}, \sigma$ are the constants from Theorem 2.1 and Lemma 2.2

Observe that nowhere integrability of $H$ implies that for every $p \in M$, the function $q \mapsto d_{H}(p, q)$ is continuous with respect to the Riemannian distance dist. By compactness of $M$, it follows that $q \mapsto d_{H}(p, q)$ is in fact uniformly continuous relative to dist. Therefore, there exists $\eta>0$ such that for all $p, q \in M, d_{H}(p, q)<$ $\tau / 2$, whenever $\operatorname{dist}(p, q)<\eta$.

Set

$$
\varrho=\min \left(\eta, \frac{\tau}{2}, \frac{1}{2^{\frac{1+\theta}{\theta}} c_{M} K^{1 / \theta}\left(\sin \phi_{0}\right)^{-1 / \theta}\|\alpha\|_{C^{\theta}}^{1 / \theta}}\right) .
$$

Observe that if $\operatorname{dist}(x, y)<\varrho$, then $\operatorname{dist}(x, y)+d_{H}(x, y)<\tau$.

Take any two points $p, q$ on $\gamma$ satisfying $\operatorname{dist}(p, q)<\varrho$. Denote the segment of $\gamma$ starting at $p$ and ending at $q$ by $\gamma_{0}$. Let $\varepsilon>0$ be arbitrary but sufficiently small so that $\operatorname{dist}(p, q)+d_{H}(p, q)+\varepsilon<\delta_{M}$. Finally, let $\gamma_{1}$ be a horizontal path from $p$ to $q$ with $\left|\gamma_{1}\right|<d_{H}(p, q)+\varepsilon$. (Note that a subriemannian geodesic from $p$ to $q$ may not exist, since $H$ is assumed to be only Hölder.) Define $\Gamma=\gamma_{0}-\gamma_{1}$; $\Gamma$ is a closed piecewise $C^{1}$ path.

Since

$$
|\Gamma|=\left|\gamma_{0}\right|+\left|\gamma_{1}\right|<\operatorname{dist}(p, q)+d_{H}(p, q)+\varepsilon<\tau<\delta_{M},
$$

by Corollary 2.3 there exists a 2-disk $D$ such that $\partial D=\Gamma$ and $|D| \leq c_{M}|\Gamma|^{2}$ (see Figure 21). On the other hand, since $\tau<\sigma$, it follows that $|\partial D|=|\Gamma|<\sigma$. Hence we can apply Theorem 2.1 to $\alpha$ on $D$. Observe that along $\gamma_{0}$, we have $\alpha_{\gamma}=(\sin \phi)^{-1} \alpha \leq\left(\sin \phi_{0}\right)^{-1} \alpha$. Along $\gamma_{1}$, both $\alpha$ and $\alpha_{\gamma}$ are zero, so the inequality 


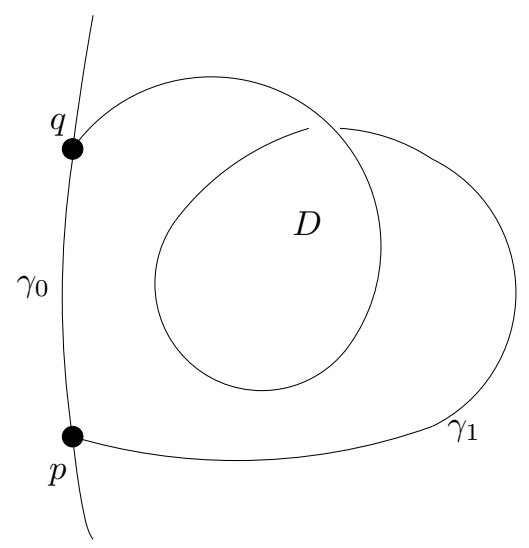

FiguRE 2. Vertical path $\gamma_{0}$ and horizontal path $\gamma_{1}$.

$\alpha_{\gamma} \leq\left(\sin \phi_{0}\right)^{-1} \alpha$ still holds. Therefore:

$$
\begin{aligned}
\left|\gamma_{0}\right| & =\int_{\partial D} \alpha_{\gamma} \\
& \leq\left(\sin \phi_{0}\right)^{-1} \int_{\partial D} \alpha \\
& \leq K\left(\sin \phi_{0}\right)^{-1}\|\alpha\|_{C^{\theta}}|\partial D|^{1-\theta}|D|^{\theta} \\
& \leq K\left(\sin \phi_{0}\right)^{-1}\|\alpha\|_{C^{\theta}}|\partial D|^{1-\theta}\left(c_{M}|\partial D|^{2}\right)^{\theta} \\
& =c_{M}^{\theta} K\left(\sin \phi_{0}\right)^{-1}\|\alpha\|_{C^{\theta}}|\partial D|^{1+\theta} \\
& =c_{M}^{\theta} K\left(\sin \phi_{0}\right)^{-1}\|\alpha\|_{C^{\theta}}\left(\left|\gamma_{0}\right|+\left|\gamma_{1}\right|\right)^{1+\theta} \\
& <c_{M}^{\theta} K\left(\sin \phi_{0}\right)^{-1}\|\alpha\|_{C^{\theta}}\left\{\left|\gamma_{0}\right|+d_{H}(p, q)+\varepsilon\right\}^{1+\theta} .
\end{aligned}
$$

Taking the $(1+\theta)$-th root of each side and regrouping, we obtain

$$
\begin{gathered}
\left|\gamma_{0}\right|^{\frac{1}{1+\theta}}-c_{M}^{\frac{\theta}{1+\theta}} K^{\frac{1}{1+\theta}}\left(\sin \phi_{0}\right)^{-\frac{1}{1+\theta}}\|\alpha\|_{C^{\theta}}^{\frac{1}{1+\theta}}\left|\gamma_{0}\right|<c_{M}^{\frac{\theta}{1+\theta}} K^{\frac{1}{1+\theta}} \\
\times\left(\sin \phi_{0}\right)^{-\frac{1}{1+\theta}}\|\alpha\|_{C^{\theta}}^{\frac{1}{1+\theta}}\left\{d_{H}(p, q)+\varepsilon\right\} .
\end{gathered}
$$

Factoring $\left|\gamma_{0}\right|^{\frac{1}{1+\theta}}$ out, the left-hand side becomes

$$
\left|\gamma_{0}\right|^{\frac{1}{1+\theta}}\left\{1-c_{M}^{\frac{\theta}{1+\theta}} K^{\frac{1}{1+\theta}}\left(\sin \phi_{0}\right)^{-\frac{1}{1+\theta}}\|\alpha\|_{C^{\theta}}^{\frac{1}{1+\theta}}\left|\gamma_{0}\right|^{\frac{\theta}{1+\theta}}\right\} .
$$

It is not hard to see that the assumption $\left|\gamma_{0}\right|=\operatorname{dist}(p, q)<\varrho$ implies that the quantity in the curly braces is $\geq 1 / 2$. Therefore,

$$
\frac{1}{2}\left|\gamma_{0}\right|^{\frac{1}{1+\theta}}<c_{M}^{\frac{\theta}{1+\theta}} K^{\frac{1}{1+\theta}}\left(\sin \phi_{0}\right)^{-\frac{1}{1+\theta}}\|\alpha\|_{C^{\theta}}^{\frac{1}{1+\theta}}\left\{d_{H}(p, q)+\varepsilon\right\} .
$$

Since $\varepsilon>0$ can be arbitrarily small and $\left|\gamma_{0}\right|=\operatorname{dist}(p, q)$, we obtain

$$
\operatorname{dist}(p, q)^{\frac{1}{1+\theta}} \leq 2 c_{M}^{\frac{\theta}{1+\theta}} K^{\frac{1}{1+\theta}}\left(\sin \phi_{0}\right)^{-\frac{1}{1+\theta}}\|\alpha\|_{C^{\theta}}^{\frac{1}{1+\theta}} d_{H}(p, q) .
$$


This completes the proof with

$$
C=\left\{2 c_{M}^{\frac{\theta}{1+\theta}} K^{\frac{1}{1+\theta}}\left(\sin \phi_{0}\right)^{-\frac{1}{1+\theta}}\|\alpha\|_{C^{\theta}}^{\frac{1}{1+\theta}}\right\}^{-1} .
$$

Remark. (a) It follows from [Sim09] that $K$ stays bounded as $\theta \rightarrow 1-$. Therefore, by (5), the constant $C=C(\theta)$ does not blow up as $\theta \rightarrow 1-$. This implies that if $H$ is $C^{1}$ (hence $C^{\theta}$, for all $0<\theta<1$ ), we recover the old quadratic estimate by letting $\theta \rightarrow 1-$.

(b) Note also that as the angle between $\gamma$ and $H$ goes to zero (i.e., as $\gamma$ tends to a horizontal path), $C \rightarrow 0$. This can be interpreted as follows: along horizontal paths, $d_{H}$ and dist are equivalent, so (3) cannot hold for any positive $C$.

The following question appears to be much harder than the one investigated in this paper:

Question. Is there an analogous upper bound for $d_{H}$ with respect to dist when $H$ is $C^{\theta}$ ?

This and essentially all other questions of Hölder subriemannian geometry remain wide open.

\section{REFERENCES}

[DW03] D. Dolgopyat and A. Wilkinson, Stable accessibility is $C^{1}$ dense, Astérisque XVII (2003), no. 287, 33-60. MR2039999 (2005d:37058)

[Gro83] Mikhail Gromov, Filling Riemannian manifolds, J. Diff. Geometry 18 (1983), 1-147. MR697984(85h:53029)

[Gro96] Carnot-Carathéodory spaces seen from within, Sub-Riemannian Geometry (A. Bellaiche and J.-J. Risler, eds.), Progress in Mathematics, vol. 144, Birkhäuser, 1996, pp. 79-323.

[HPS77] Morris W. Hirsch, Charles C. Pugh, and Michael Shub, Invariant manifolds, Lecture Notes in Mathematics, vol. 583, Springer-Verlag, Berlin-New York, 1977. MR0501173 $(58: 18595)$

[Mon02] Richard Montgomery, A tour of subriemannian geometries, their geodesics and applications, Mathematical Surveys and Monographs, vol. 91, Amer. Math. Soc., Providence, RI, 2002. MR:1867362 (2002m:53045)

[Pes04] Yakov B. Pesin, Lectures on partial hyperbolicity and stable ergodicity, Zurich Lectures in Advanced Mathematics, European Mathematical Society (EMS), Zürich, 2004. MR2068774 (2005j:37039)

[PS04] Charles C. Pugh and Michael Shub, Stable ergodicity, Bull. Amer. Math. Soc. (N.S.) 41 (2004), 1-41. MR2015448(2005f:37011)

[Sim09] Slobodan N. Simić, Hölder forms and integrability of invariant distributions, Discrete Contin. Dyn. Syst. 25 (2009), no. 2, 669-685. MR2525198

Department of Mathematics, San José State University, San José, California 951920103

E-mail address: simic@math.sjsu.edu 